PROCEEDINGS OF THE

AMERICAN MATHEMATICAL SOCIETY

Volume 109, Number 4, August 1990

\title{
THE LIE ALGEBRA ASSOCIATED TO THE LOWER CENTRAL SERIES OF A LINK GROUP AND MURASUGI'S CONJECTURE
}

\author{
JOHN P. LABUTE
}

(Communicated by Frederick R. Cohen)

\begin{abstract}
The Chen-Milnor presentation can be used to determine the Lie Algebra associated to the lower central series of the fundamental group of a link in the 3-sphere $S^{3}$ in many interesting cases. We use this fact to obtain new and simpler proofs of unpublished results of Maeda on a conjecture of Murasugi in the sharpened form of Massey and Traldi.
\end{abstract}

\section{STATEMENT OF RESULTS}

Let $L$ be a link in $S^{3}$ with $m$ components and let $G=\pi_{1}\left(S^{3}-L\right)$ be the group of the link. Let $F=F(m)$ be the free group on $x_{1}, \ldots, x_{m}$ and let $F_{n}$ denote the $n$th term of the lower central series of $F$. By a result of Milnor [9, Theorem 4] there are, for each integer $q \geq 1$, elements $w_{1}^{(q)}, \ldots, w_{m}^{(q)} \in F$ such that $w_{i}^{(q+1)} \equiv w_{i}^{(q)}$ modulo $F_{q+1}$ and such that $G / G_{q+1}$ has the presentation

$$
\left\langle x_{1}, \ldots, x_{m}:\left[x_{1}, w_{1}^{(q)}\right]=\cdots\left[x_{m}, w_{m}^{(q)}\right]=1, F_{q+1}=1\right\rangle
$$

where $[x, y]=x^{-1} y^{-1} x y$. Moreover, for any $q \geq 1$, any one of the relators $r_{i}^{(q)}=\left[x_{i}, w_{i}^{(q)}\right]$ is redundant. Let $R^{(q)}$ be the normal subgroup of $F$ generated by $r_{1}^{(q)}, \ldots, r_{m}^{(q)}$.

Let $\mathfrak{L}=\mathfrak{L}(F)=\bigoplus_{q>1} \mathfrak{L}_{q}(F)$ be the Lie algebra associated to the lower central series of $F$. If $\xi_{i}$ is the image of $x_{i}$ in $\mathfrak{L}_{1}$ then $\mathfrak{L}$ is the free Lie algebra over $\mathbf{Z}$ on $\xi_{1}, \ldots, \xi_{m}$. Let $\mathfrak{g}=\mathfrak{L}(G)$ be the Lie algebra associated to the lower central series of $G$. If $\mathfrak{g}^{(q)}=\mathfrak{L}\left(F / R^{(q)} F_{q+1}\right)$ then $\mathfrak{g}^{(q)}=$ $\bigoplus_{1 \leq i \leq q} \mathfrak{g}_{i}=\mathfrak{L}\left(G / G_{q+1}\right)$. Since $\mathfrak{g}^{(q+1)}=\mathfrak{g}^{(q)} \oplus \mathfrak{L}_{q+1}\left(F / R^{(q+1)} F_{q+2}\right)$, we see that $\mathfrak{g}=\bigoplus_{q \geq 1} \mathfrak{L}_{q}\left(F / R^{(q)} F_{q+1}\right)$. Hence $\mathfrak{g}=\mathfrak{L} / \mathfrak{R}$ where $\mathfrak{R}=\bigoplus_{q \geq 1} \mathfrak{R}_{q}$ with $\mathfrak{R}_{q}=\operatorname{Ker}\left(\mathfrak{L}_{q}(F) \rightarrow \mathfrak{L}_{q}\left(F / R^{(q)} F_{q+1}\right)\right)$. The ideal $\mathfrak{R}$ is in general very difficult to determine. However, generators for it can be given if the relators $r_{i}^{(q)}$ satisfy a certain independence condition, which we now describe.

Received by the editors December 5, 1989 and, in revised form, September 6, 1989; presented at the Canadian Mathematical Society Winter Meeting, Vancouver, BC, December 1987.

1980 Mathematics Subject Classification (1985 Revision). Primary 20F40, 57M25.

Supported by Canada NSERC Grant A4344 and Quebec FCAC Grant 88-EQ-2489. 
Without loss of generality we may assume that $r_{i}^{(q)} \neq 1$ for $1 \leq i \leq s$ and $q$ sufficiently large while $r_{i}^{(q)}=1$ for $i>s, q \geq 1$. Then, for $1 \leq i \leq s$ there are integers $d_{i}, q_{i}$ such that for $q \geq q_{i}$ we have $r_{i}^{(q)} \in F_{d_{i}}$ but $\notin F_{d_{i}+1}$. For $q \geq q_{i}$ the image of $r_{i}^{(q)}$ in $\mathfrak{L}_{d_{i}}$ is independent of $q$ and is denoted by $\rho_{i}$. Also one can assume that $\rho_{s}$ is a linear combination of $\rho_{1}, \ldots, \rho_{s-1}$, cf. [10, p. 295]. Let $\mathfrak{r}$ be the ideal of $\mathfrak{L}$ generated by $\rho_{1}, \ldots, \rho_{s-1}$. We have $\mathfrak{r} \subseteq \mathfrak{R}$ but this inclusion is in general proper. If $U=U(\mathfrak{L} / \mathfrak{r})$ is the enveloping algebra of $\mathfrak{L} / \mathfrak{r}$ then $\mathfrak{r} /[\mathfrak{r}, \mathfrak{r}]$ is a $U$-module via the adjoint representation of $\mathfrak{L} / \mathfrak{r}$. In [7] we show that $\mathfrak{R}=\mathfrak{r}$ if the following condition holds:

(I) $\mathfrak{L} / \mathfrak{r}$ is a free $\mathbf{Z}$-module and $\mathfrak{r} /[\mathfrak{r}, \mathfrak{r}]$ is a free $U$-module on the images of $\rho_{1}, \ldots, \rho_{s-1}$.

Following [2] and [5], we call a sequence $\rho_{1}, \ldots, \rho_{s-1}$ of elements of $\mathfrak{L}$ inert if condition (I) holds. For examples where this condition applies cf. [2], [4]. Anick [2] has shown that (I) holds if and only if, for $n \geq 1$, the $n$th homogeneous component of $\mathfrak{L} / \mathfrak{r}$ is a free $\mathbf{Z}$-module of rank $a_{n}$, where $a_{n}$ is given by $(\mathrm{A})$ :

$$
\prod_{n \geq 1}\left(1-t^{n}\right)^{a_{n}}=1-m t+\sum_{1 \leq j<s} t^{d_{j}} .
$$

If (I) holds then the inverse of the above power series is the Poincare series of the graded algebra $\operatorname{gr}(\mathbf{Z}[G])=\bigoplus_{n \geq 0} I^{n} / I^{n+1}$, where $I$ is the augmentation ideal of the group ring $\mathbf{Z}[G]$. This follows from the fact that, in this case, $\operatorname{gr}(\mathbf{Z}[G])$ is the enveloping algebra of $\mathfrak{L}(G)$, cf. [7].

Theorem 1. The following are equivalent:

(1) Property (I);

(2) For $n \geq 1, \mathfrak{L}_{n}(G)$ is a free $\mathbf{Z}$-module of rank $a_{n}$ given by (A).

Now let $L$ be a tame link in $S^{3}$ with components $K_{1}, \ldots, K_{m}$ and let $G$ be the group of the link. The linking diagram of $L$ is the edge-weighted graph $\Gamma$ whose vertices are the components of $L$, with two vertices $K_{i}$ and $K_{j}$ being joined by an edge of weight $l_{i j}=$ the linking number of $K_{i}$ with $K_{j}$. By convention, we delete from the diagram any edges of weight zero. In the above presentation of $G / G_{q+1}$, the relators $r_{i}^{(q)}$ have the form

$$
r_{i}^{(q)} \equiv \prod_{j \neq i}\left[x_{i}, x_{j}\right]^{l_{j j}} \bmod F_{3} .
$$

Assume that for each $i$ there is a $j$ such that $l_{i j} \neq 0$, i.e., that each initial form $\rho_{i}$ is of degree 2 . Then

$$
\rho_{i}=\sum_{j \neq i} l_{i j}\left[\xi_{i}, \xi_{j}\right]
$$

The conjecture of Murasugi we are dealing with concerns the property (M).

$$
\mathfrak{L}_{n}(G) \cong \mathfrak{L}_{n}(F(m-1)) \text { for } n \geq 2 \text {. }
$$


His conjecture was that property (M) held if $l_{i j}= \pm 1$ for all $i, j$. In [8] Maeda proved the following sharpened form of this conjecture: property $(\mathbf{M})$ holds if $\Gamma$ has a spanning subtree whose edges have weights \pm 1 . His proof uses the Chen groups $C_{n}(G)=G_{n} G^{\prime \prime} / G_{n+1} G^{\prime \prime}$, where $G^{\prime}=[G, G]$ is the derived group of $G$ and $G^{\prime \prime}$ is the second derived group of $G$. He also shows that property $(\mathbf{M})$ is equivalent to $(\widetilde{\mathbf{M}})$ :

$$
C_{n}(G) \cong C_{n}(F(m-1)) \text { for } n \geq 2 .
$$

Using this, Massey and Traldi [9] were able to show that $(\widetilde{\mathbf{M}})$ was equivalent to the statement that $G_{2} / G_{3} \cong H_{2} / H_{3}$, where $H=F(m-1)$. In this paper we give a completely independent proof of this result that avoids the use of Chern groups.

Theorem 2. The following are equivalent:

(1) The linking diagram $\Gamma$ is connected $\bmod p$ for every prime $p$;

(2) Property (I) holds with $s=m$ and $d_{1}=\cdots=d_{s-1}=2$;

(3) Property (M);

(4) The group $\mathfrak{L}_{2}(G)$ is a free $\mathbf{Z}$-module of rank $(m-1)(m-2) / 2$.

The graph $\Gamma$ is said to be connected modulo $p$ if there is a spanning subtree of $\Gamma$ whose edges have weights that are not congruent to zero modulo $p$. This theorem sharpens Theorem 3.7 of [2].

Corollary (Massey-Traldi). Let $G$ be the group of an m-component link in $S^{3}$. Then, for all primes $p$, the rank of $\mathfrak{L}_{2}(G) \otimes_{\mathbf{Z}} \mathbf{F}_{p}$ is greater than or equal to $(m-1)(m-2) / 2$ with equality for all primes $p$ if and only if the equivalent conditions of Theorem 2 hold.

This result follows immediately from the fact that $\mathfrak{L}_{2}(G)$ is the quotient of $\mathfrak{L}_{2}(F)$ by the subgroup generated by those $\rho_{i}$, which are of degree 2 .

Proof of Theorem 1. It remains only to prove that (2) implies (1). So assume that (2) holds and let $U$ be the enveloping algebra of $\mathfrak{L} / \mathfrak{r}$. Let $V$ be the free $U$ module on generators $v_{1}, \ldots, v_{s-1}$ with $v_{i}$ of degree $d_{i}$ and let $M=\mathfrak{r} /[\mathfrak{r}, \mathfrak{r}]$. Let $\gamma: V \rightarrow M$ be the $U$-module homomorphism with $\gamma\left(v_{i}\right)$ equal to the image of $\rho_{i}$ in $M$. Then (I) holds if and only if $U$ is $Z$-free and $\gamma$ is bijective.

Now let $p$ be a prime, let $\overline{\mathfrak{L}}=\mathfrak{L} \otimes \mathbf{F}_{p}$, let $\bar{\rho}_{i}$ be the image of $\rho_{i}$ in $\overline{\mathfrak{L}}$, let $\overline{\mathfrak{r}}$ be the ideal of $\overline{\mathfrak{L}}$ generated by $\bar{\rho}_{1}, \ldots, \bar{\rho}_{s-1}$ and let $\bar{U}$ be the enveloping algebra of $\overline{\mathfrak{L}} / \overline{\mathfrak{r}}$. If $\bar{V}=V \otimes \mathbf{F}_{p}$ and $\bar{M}=\overline{\mathfrak{r}} /[\overline{\mathfrak{r}}, \overline{\mathfrak{r}}]$, let $\bar{\gamma}: \bar{V} \rightarrow \bar{M}$ be the $\bar{U}$ module homomorphism sending $\bar{v}_{i}=v_{i} \otimes 1$ to the image of $\bar{\rho}_{i}$ in $\bar{M}$. Then (I) holds if and only if $\bar{\gamma}$ is bijective for every prime $p$.

Let $N(t)$ denote the Poincare series of a graded vector space $N$. If $\bar{W}$ is the enveloping algebra of $\overline{\mathfrak{r}}$ we have $\bar{W}(t)=(1-\bar{M}(t))^{-1}$ and, if $\bar{K}$ is the kernel of $\bar{\gamma}$ we have

$$
\bar{M}(t)=\left(t^{d_{1}}+\cdots+t^{d_{s-1}}\right) \bar{U}(t)+\bar{K}(t) .
$$


Using the fact that $\bar{U}(t) \bar{W}(t)=(1-m t)^{-1}$, we obtain

$$
\bar{U}(t)^{-1}=1-m t+\sum_{1 \leq i<s} t^{d_{i}}+\bar{K}(t) \bar{U}(t)^{-1} .
$$

It follows that $\bar{\gamma}$ is bijective in degrees $\leq n$ if and only if

$$
\bar{U}(t) \equiv\left(1-m t+\sum_{1 \leq i<s} t^{d_{i}}\right)^{-1} \bmod t^{n+1}
$$

Call $\rho_{1}, \ldots, \rho_{s-1} n$-inert $($ resp. $n$-inert $\bmod p)$ if $\gamma($ resp. $\bar{\gamma})$ is bijective in degrees $\leq n$. Then, since the sequence $\rho_{1}, \ldots, \rho_{s-1}$ is $n$-inert if and only if it is inert $\bmod p$ for every prime $p$, we see that the sequence $\rho_{1}, \ldots, \rho_{s-1}$ is $n$-inert if and only if $(\mathrm{X})$ holds for every prime $p$.

Now suppose that $\rho_{1}, \ldots, \rho_{s-1}$ is $n$-inert for some $n$ (this is true for $n=$ $0)$. Then by the proof of $[7$, Theorem 1, p. 54] we obtain that $\mathfrak{L} / \mathfrak{R}$ equals $\mathfrak{L} / \mathfrak{r}$ in degrees $\leq n+1$. Since (2) holds we obtain $(\mathrm{X})$ with $n$ replaced by $n+1$, i.e., that $\rho_{1}, \ldots, \rho_{s-1}$ is $(n+1)$-inert. Thus $\rho_{1}, \ldots, \rho_{s-1}$ is $n$-inert for all $n$, which is Property (I).

Proof of Theorem 2. The implication $(1) \Rightarrow(2)$ is a result of Anick [2, Proposition 3.5].

If (2) holds then we have shown in [7] that $\mathfrak{r}=\mathfrak{R}$ and hence that $\mathfrak{L}(G)=$ $\mathfrak{L} / \mathfrak{r}$. By Anick [2, Theorem 1.6], or by results of [2] in conjunction with [4], the enveloping algebra of $\mathfrak{L}(G)$ is a free $\mathbf{Z}$-module whose $n$th homogeneous component is of rank equal to the coefficient of $t^{n}$ in the formal power series

$$
\left(1-m t+(m-1) t^{2}\right)^{-1}=((1-t)(1-(m-1) t))^{-1}
$$

The right-hand side of $(F)$ is the Poincare series for the enveloping algebra of $\mathfrak{L}(F(1)) \oplus \mathfrak{L}(F(m-1))$, hence $\mathfrak{L}(G) \cong \mathfrak{L}(F(1)) \oplus \mathfrak{L}(F(m-1))$ as graded abelian groups. Since $\mathfrak{L}_{n}(F(1))=0$ for $n \geq 2$, Property (M) follows. That (3) implies (4) is immediate.

To show that (4) implies (1) let $\Lambda=\left(\lambda_{i(j, k)}\right)$ be the $m \times m(m-1) / 2$ matrix, whose rows are indexed by $1, \ldots, m$ and whose columns are indexed by the pairs $(i, j)$ with $1 \leq i<j \leq m$, with $\lambda_{i(i, j)}=l_{i j}, \lambda_{j(i, j)}=-l_{i j}$ and $\lambda_{i(j, k)}=0$ otherwise. Then, since $\Lambda$ is a presentation matrix for the abelian group $\mathfrak{L}_{2}(G)$, we see that (4) holds if and only if for every prime $p$ the reduction of $\Lambda \bmod p$ has rank $m-1$. Since the $(i, j)$ th column of $\Lambda$ is $l_{i j}$ times a column vector with entries equal to zero except in the $i$ th and $j$ th rows where the entries are 1 and -1 respectively, we see that the linking diagram of $L$ is connected $\bmod p$ if and only if the reduction of $\Lambda \bmod p$ has $\operatorname{rank} m-1$. 
Remarks. In [8] Maeda uses the following independence condition:

(MI) $\mathfrak{L}^{\prime} / \mathfrak{r}+\mathfrak{L}^{\prime \prime}$ is a free Z-module and the quotient $\mathfrak{r} / \mathfrak{r} \cap \mathfrak{L}^{\prime \prime}$ is a free $U\left(\mathfrak{L} / \mathfrak{L}^{\prime}\right)$ module on the images of $\rho_{1}, \ldots, \rho_{s-1}$.

Here $\mathfrak{L}^{\prime}=[\mathfrak{L}, \mathfrak{L}]$ is the derived algebra of $\mathfrak{L}$ and $\mathfrak{L}^{\prime \prime}$ is the second derived algebra. His main result is to show that (MI) implies that $\mathfrak{R}=\mathfrak{r}$ and that $\mathfrak{L}(G)$ is Z-free. However he does not give a formula for the rank of $\mathfrak{L}_{n}(G)$ except in the case each $\rho_{i}$ is of degree 2, which is enough to settle Murasugi's conjecture. This he does by giving an algorithm for getting a basis of $\mathfrak{L}_{n}(G)$ that shows that the rank in question depends only on the degrees of the $\rho_{i} \mathrm{~s}$ and their multiplicities. He is therefore reduced to computing the rank in a special case, which he does in the case of degree 2.

We finish by showing that (MI) implies (I). Since the sequence

$$
0 \rightarrow \mathfrak{r} / \mathfrak{r} \cap \mathfrak{L}^{\prime \prime}+\mathfrak{L}^{\prime} / \mathfrak{L}^{\prime \prime} \rightarrow \mathfrak{L}^{\prime} / \mathfrak{r}+\mathfrak{L}^{\prime \prime} \rightarrow 0
$$

is exact, we see that (MI) holds if and only if the elements

$$
\operatorname{ad}\left(\xi_{1}\right)^{i_{1}} \cdots \operatorname{ad}\left(\xi_{m}\right)^{i_{m}}\left(\rho_{j}\right), \quad i_{k} \geq 0,1 \leq j \leq s-1,
$$

are part of a basis for the graded free Z-module $\mathfrak{L}^{\prime} / \mathfrak{L}^{\prime \prime}$ and hence part of a basis of the free Lie algebra $\mathfrak{L}^{\prime}$, cf. [6, Prop. 2]. The elements $(*)$ generate $\mathfrak{r}$ as an ideal of $\mathfrak{L}^{\prime}$ and hence $\mathfrak{r} /[\mathfrak{r}, \mathfrak{r}]$ is a free $U\left(\mathfrak{L}^{\prime} / \mathfrak{r}\right)$-module on their images in $\mathfrak{r} /[\mathfrak{r}, \mathfrak{r}]$, cf. [3, Prop. 10]. Since $U\left(\mathfrak{L} / \mathfrak{L}^{\prime}\right)$ is the polynomial ring over $\mathbf{Z}$ on $\xi_{1}, \ldots, \xi_{m}$ it follows that $\mathfrak{r} /[\mathfrak{r}, \mathfrak{r}]$ is a free $U(\mathfrak{L} / \mathfrak{r})$-module on the images of $\rho_{1}, \ldots, \rho_{s-1}$. Also, $\mathfrak{L} / \mathfrak{r}$ is $\mathbf{Z}$-free since $\mathfrak{L} / \mathfrak{L}^{\prime}$ is $\mathbf{Z}$-free and $\mathfrak{L}^{\prime} / \mathfrak{r}$ is a free Lie algebra over $\mathbf{Z}$. Hence (I) holds.

From the above proof one also deduces that (MI) implies that the derived algebra of $\mathfrak{L}(G)$ is a free Lie algebra over $\mathbf{Z}$.

\section{ACKNOWLEDGMENT}

We would like to thank L. Traldi for his helpful comments on the ChenMilnor presentation of a link and the referee for his elegant proof of Theorem 1.

\section{REFERENCES}

1. D. Anick, Non-commutative graded algebras and their Hilbert series, J. Algebra 78 (1982), 120-140.

2. __ Inert sets and the Lie algebra associated to a group, J. Algebra 111 (1987), 154-165.

3. N. Bourbaki, Groupes et algèbres de Lie, Hermann, Paris, 1972, Chapter 2.

4. R. Hain, Iterated integrals, intersection theory and link groups, Topology 24 (1985), 45-66.

5. S. Halperin and J.-M. Lemaire, Suites insertes dans les algèbres de Lie graduée, Math. Scand. 61 (1987), 39-67.

6. J. P. Labute, Algèbres de Lie et pro-p-groupes définis par une seule relation, Invent. Math. 4 (1967), 142-158.

7. Trans. Amer. Math. Soc. 288 (1985), 51-57. 
8. T. Maeda, Lower central series of link groups, Ph.D. thesis, Univ. of Toronto (1983), 1-104.

9. W. S. Massey and L. Traldi, On a conjecture of K. Murasugi, Pacific J. Math. 124 (1986), 193-213.

10. J. Milnor, Isotopy of links, Algebraic Geometry and Topology, A Symposium in Honor of S. Lefschetz, Princeton University Press, Princeton, NJ, pp. 280-306.

Department of Mathematics, McGill University, Montreal, Quebec H3A 2K6 CANADA 